MATHEMATICS OF COMPUTATION

Volume 77, Number 262, April 2008, Pages 1057-1074

S 0025-5718(07)01968-0

Article electronically published on October 24, 2007

\title{
THE ABEL LEMMA AND THE $q$-GOSPER ALGORITHM
}

\author{
VINCENT Y. B. CHEN, WILLIAM Y. C. CHEN, AND NANCY S. S. GU
}

\begin{abstract}
Chu has recently shown that the Abel lemma on summation by parts reveals the telescoping nature of Bailey's $6 \psi_{6}$ bilateral summation formula. We present a systematic approach to compute Abel pairs for bilateral and unilateral basic hypergeometric summation formulas by using the $q$-Gosper algorithm. It is demonstrated that Abel pairs can be derived from Gosper pairs. This approach applies to many classical summation formulas.
\end{abstract}

\section{INTRODUCTION}

We follow the notation and terminology in $[10$. For $|q|<1$, the $q$-shifted factorial is defined by

$$
(a ; q)_{\infty}=\prod_{k=0}^{\infty}\left(1-a q^{k}\right) \text { and }(a ; q)_{n}=\frac{(a ; q)_{\infty}}{\left(a q^{n} ; q\right)_{\infty}}, \text { for } n \in \mathbb{Z} .
$$

For convenience, we shall adopt the following notation for multiple $q$-shifted factorials:

$$
\left(a_{1}, a_{2}, \ldots, a_{m} ; q\right)_{n}=\left(a_{1} ; q\right)_{n}\left(a_{2} ; q\right)_{n} \cdots\left(a_{m} ; q\right)_{n},
$$

where $n$ is an integer or infinity. In particular, for a nonnegative integer $k$, we have

$$
(a ; q)_{-k}=\frac{1}{\left(a q^{-k} ; q\right)_{k}} .
$$

The (unilateral) basic hypergeometric series ${ }_{r} \phi_{s}$ is defined by

$$
{ }_{r} \phi_{s}\left[\begin{array}{cccc}
a_{1}, & a_{2}, & \ldots, & a_{r} \\
b_{1}, & b_{2}, & \ldots, & b_{s}
\end{array} ;, z\right]=\sum_{k=0}^{\infty} \frac{\left(a_{1}, a_{2}, \ldots, a_{r} ; q\right)_{k}}{\left(q, b_{1}, b_{2}, \ldots, b_{s} ; q\right)_{k}}\left[(-1)^{k} q^{\left(\begin{array}{c}
k \\
2
\end{array}\right)}\right]^{1+s-r} z^{k},
$$

while the bilateral basic hypergeometric series ${ }_{r} \psi_{s}$ is defined by

$$
{ }_{r} \psi_{s}\left[\begin{array}{cccc}
a_{1}, & a_{2}, & \ldots, & a_{r} \\
b_{1}, & b_{2}, & \ldots, & b_{s}
\end{array} ;, z\right]=\sum_{k=-\infty}^{\infty} \frac{\left(a_{1}, a_{2}, \ldots, a_{r} ; q\right)_{k}}{\left(b_{1}, b_{2}, \ldots, b_{s} ; q\right)_{k}}\left[(-1)^{k} q^{\left(\begin{array}{c}
k \\
2
\end{array}\right)}\right]^{s-r} z^{k} .
$$

Recently Chu [9] used the Abel lemma on summation by parts to give an elementary proof of Bailey's very well-poised ${ }_{6} \psi_{6}$-series identity [5] (see also, [10,

Received by the editor July 26, 2006 and, in revised form, August 2, 2006.

2000 Mathematics Subject Classification. Primary 33D15; Secondary 33F10.

Key words and phrases. The Abel lemma, Abel pairs, basic hypergeometric series, the $q$-Gosper algorithm, Gosper pairs.

This work was supported by the 973 Project on Mathematical Mechanization, the Ministry of Education, the Ministry of Science and Technology, and the National Science Foundation of China.

(C)2007 American Mathematical Society Reverts to public domain 28 years from publication 
Appendix II.33]):

$$
\begin{aligned}
& { }_{6} \psi_{6}\left[\begin{array}{cccccc}
q a^{\frac{1}{2}}, & -q a^{\frac{1}{2}}, & b, & c, & d, & e \\
a^{\frac{1}{2}}, & -a^{\frac{1}{2}}, & a q / b, & a q / c, & a q / d, & a q / e
\end{array} ; q, \frac{q a^{2}}{b c d e}\right] \\
& =\frac{(q, a q, q / a, a q / b c, a q / b d, a q / b e, a q / c d, a q / c e, a q / d e ; q)_{\infty}}{\left(a q / b, a q / c, a q / d, a q / e, q / b, q / c, q / d, q / e, q a^{2} / b c d e ; q\right)_{\infty}},
\end{aligned}
$$

where $\left|q a^{2} / b c d e\right|<1$.

The Abel lemma on summation by parts is stated as

$$
\sum_{k=-\infty}^{\infty} A_{k}\left(B_{k}-B_{k-1}\right)=\sum_{k=-\infty}^{\infty} B_{k}\left(A_{k}-A_{k+1}\right)
$$

provided that the series on both sides are convergent and $\sum_{k} A_{k} B_{k}$ is absolutely convergent. Based on the Abel lemma, Chu found an Abel pair $\left(A_{k}, B_{k}\right)$ :

$$
\begin{aligned}
A_{k} & =\frac{\left(b, c, d, q^{2} a^{2} / b c d ; q\right)_{k}}{(a q / b, a q / c, a q / d, b c d / a q ; q)_{k}}, \\
B_{k} & =\frac{(q e, b c d / a ; q)_{k}}{\left(a q / e, q^{2} a^{2} / b c d ; q\right)_{k}}\left(\frac{q a^{2}}{b c d e}\right)^{k},
\end{aligned}
$$

which leads to the following iteration relation:

$$
\begin{aligned}
\Omega(a ; b, c, d, e)= & \Omega(a q ; b, c, d, e q) \\
& \times \frac{a(1-e)(1-a q)(1-a q / b c)(1-a q / b d)(1-a q / c d)}{e(1-a)(1-a q / b)(1-a q / c)(1-a q / d)\left(1-a^{2} q / b c d e\right)},
\end{aligned}
$$

where

$$
\Omega(a ; b, c, d, e)={ }_{6} \psi_{6}\left[\begin{array}{cccccc}
q a^{\frac{1}{2}}, & -q a^{\frac{1}{2}}, & b, & c, & d, & e \\
a^{\frac{1}{2}}, & -a^{\frac{1}{2}}, & a q / b, & a q / c, & a q / d, & a q / e
\end{array} ; q, \frac{q a^{2}}{b c d e}\right] .
$$

Because of the symmetries in $b, c, d$, and $e$, applying the identity (1.4) three times with respect to the parameters $a$ and $d, a$ and $c, a$ and $b$, we arrive at the following iteration relation:

$$
\begin{aligned}
\Omega(a ; b, c, d, e)= & \Omega\left(a q^{4} ; b q, c q, d q, e q\right) \\
& \times \frac{a^{4} q^{6}}{b c d e} \cdot \frac{1-a q^{4}}{1-a} \cdot \frac{(1-b)(1-c)(1-d)(1-e)}{\left(a^{2} q / b c d e ; q\right)_{4}} \\
& \times \frac{(a q / b c, a q / b d, a q / b e, a q / c d, a q / c e, a q / d e ; q)_{2}}{(a q / b, a q / c, a q / d, a q / e ; q)_{3}} .
\end{aligned}
$$

Again, iterating the above relation $m$ times, we get

$$
\begin{aligned}
\Omega(a ; b, c, d, e)= & \Omega\left(a q^{4 m} ; b q^{m}, c q^{m}, d q^{m}, e q^{m}\right) \\
& \times \frac{a^{4 m} q^{6 m^{2}}}{(b c d e)^{m}} \cdot \frac{1-a q^{4 m}}{1-a} \cdot \frac{(b, c, d, e ; q)_{m}}{\left(a^{2} q / b c d e ; q\right)_{4 m}} \\
& \times \frac{(a q / b c, a q / b d, a q / b e, a q / c d, a q / c e, a q / d e ; q)_{2 m}}{(a q / b, a q / c, a q / d, a q / e ; q)_{3 m}} .
\end{aligned}
$$


Replacing the summation index $k$ by $k-2 m$, we obtain the transformation formula:

$$
\begin{aligned}
\Omega(a ; b, c, d, e)= & \Omega\left(a ; b q^{-m}, c q^{-m}, d q^{-m}, e q^{-m}\right) \\
& \times \frac{(a q / b c, a q / b d, a q / b e, a q / c d, a q / c e, a q / d e ; q)_{2 m}}{(q / b, q / c, q / d, q / e, a q / b, a q / c, a q / d, a q / e ; q)_{m}\left(a^{2} q / b c d e ; q\right)_{4 m}}
\end{aligned}
$$

Setting $m \rightarrow \infty$, Chu obtained the ${ }_{6} \psi_{6}$ summation formula (1.2) by Jacobi's triple product identity [10, Appendix II.28]:

$$
\sum_{k=-\infty}^{\infty} q^{k^{2}} z^{k}=\left(q^{2},-q z,-q / z ; q^{2}\right)_{\infty}
$$

since

$$
\begin{aligned}
& \lim _{m \rightarrow \infty} \Omega\left(a ; b q^{-m}, c q^{-m}, d q^{-m}, e q^{-m}\right) \\
& =\lim _{m \rightarrow \infty}{ }_{6} \psi_{6}\left[\begin{array}{cccccc}
q a^{\frac{1}{2}}, & -q a^{\frac{1}{2}}, & b q^{-m}, & c q^{-m}, & d q^{-m}, & e q^{-m} \\
a^{\frac{1}{2}}, & -a^{\frac{1}{2}}, & a q^{m+1} / b, & a q^{m+1} / c, & a q^{m+1} / d, & a q^{m+1} / e
\end{array} ; \frac{a^{2} q^{4 m+1}}{b c d e}\right] \\
& =\sum_{k=-\infty}^{\infty} \frac{1-a q^{2 k}}{1-a} q^{2 k^{2}-k} a^{2 k}=\frac{1}{1-a} \sum_{k=-\infty}^{\infty}(-1)^{k} q^{\left(\begin{array}{c}
k \\
2
\end{array}\right) a^{k}}=(a q, q / a, q ; q)_{\infty} .
\end{aligned}
$$

This paper is motivated by the question of how to systematically compute the Abel pairs for bilateral summation formulas. We find that the $q$-Gosper algorithm is an efficient mechanism for this purpose. The $q$-Gosper algorithm has been extensively studied. Koorwinder gave a rigorous description of the $q$-Gosper algorithm in [14. Abramov, Paule, and Petkovšek [1] developed the package $q$ Hyper for finding all $q$-hypergeometric solutions of linear homogeneous recurrences with polynomial coefficients. Later Böing and Koepf [7] gave an algorithm for the same purpose. The Maple package $q$ sum.mpl was described by Böing and Koepf [7]. In [18, Riese presented a generalization of the $q$-Gosper algorithm to indefinite bibasic hypergeometric summations.

Recall that a function $t_{k}$ is said to be a basic hypergeometric term if $t_{k+1} / t_{k}$ is a rational function of $q^{k}$. The $q$-Gosper algorithm is devised to answer the question if there is a basic hypergeometric term $z_{k}$ for a given basic hypergeometric term $t_{k}$ such that

$$
t_{k}=z_{k+1}-z_{k} .
$$

We observe that for an iteration relation of a summation formula, the difference of the $k$ th terms of the two sides is a basic hypergeometric term so that the $q$-Gosper algorithm can be employed.

The main result of this paper is to present a general framework to deal with basic hypergeometric identities based on the $q$-Gosper algorithm. We start with an iteration relation derived from the original identity. Then we use the $q$-Gosper algorithm to generate a Gosper pair $\left(g_{k}, h_{k}\right)$ if it exists. This step can be regarded as a verification of the iteration relation. Finally, we employ the iteration relation to prove the desired identity by computing a certain limit value. In fact, once a Gosper pair $\left(g_{k}, h_{k}\right)$ is obtained, one can easily compute the corresponding Abel pair. It turns out that the Abel pair for the ${ }_{6} \psi_{6}$ sum discovered by Chu [9] coincides with the Abel pair derived from the Gopser pair by using our approach. In general, our method is efficient for many classical summation formulas with parameters. 
As examples, we give Gosper pairs and Abel pairs of several well-known bilateral summation formulas including Ramanujan's ${ }_{1} \psi_{1}$ summation formula and two formulas due to Schlosser [20. In the last section we demonstrate that the idea of Gosper pairs can be applied to unilateral summation formulas as well. We use the $q$-Gauss ${ }_{2} \phi_{1}$ summation formula as an example to illustrate the procedure to compute Gosper pairs. As another example, we derive a Gosper pair and an Abel pair for the ${ }_{6} \phi_{5}$ summation formula.

In comparison to a recent approach presented by Chen, Hou, and $\mathrm{Mu} 8$ for proving nonterminating basic hypergeometric series identities by using the $q$-Zeilberger algorithm [14, 15, 17, 22, one sees that the method in this paper does not rely on the introduction of the parameter $n$ in order to establish recurrence relations, and it only makes use of the $q$-Gosper algorithm.

\section{The GOSPER PAirs For BILATERAL SUMmations}

In spite of its innocent appearance, the Abel lemma is intrinsic for some sophisticated bilateral basic hypergeometric identities. In this section, we introduce the notion of Gosper pairs and show that one may apply the $q$-Gosper algorithm to construct Gosper pairs which can be regarded as certificates like Abel pairs to justify iteration relations for bilateral summations. Furthermore, it is easily seen that one can compute Abel pairs from Gosper pairs.

Suppose that we have a bilateral series $\sum_{-\infty}^{\infty} F_{k}\left(a_{1}, a_{2}, \ldots, a_{n}\right)$ which has a closed product form. Making the substitutions $a_{i} \rightarrow a_{i} q$ or $a_{i} \rightarrow a_{i} / q$ for some parameters $a_{i}$, the closed product formula induces an iteration relation for the summation which can be stated in the following form:

$$
\sum_{k=-\infty}^{\infty} F_{k}\left(a_{1}, a_{2}, \ldots, a_{n}\right)=\sum_{k=-\infty}^{\infty} G_{k}\left(a_{1}, a_{2}, \ldots, a_{n}\right) .
$$

A Gosper pair $\left(g_{k}, h_{k}\right)$ is a pair of basic hypergeometric terms such that

$$
\begin{aligned}
g_{k}-h_{k} & =F_{k}\left(a_{1}, a_{2}, \ldots, a_{n}\right), \\
g_{k}-h_{k+1} & =G_{k}\left(a_{1}, a_{2}, \ldots, a_{n}\right) .
\end{aligned}
$$

We assume that $\sum_{k=-\infty}^{\infty} F_{k}\left(a_{1}, a_{2}, \ldots, a_{n}\right)$ and $\sum_{k=-\infty}^{\infty} G_{k}\left(a_{1}, a_{2}, \ldots, a_{n}\right)$ are both convergent. We also assume that

$$
\lim _{k \rightarrow \infty} h_{k}=\lim _{k \rightarrow-\infty} h_{k} .
$$

Note that there are many bilateral summations with the above limit property.

Evidently, once a Gosper pair is derived, the identity (2.1) immediately follows from the telescoping relation:

$$
\sum_{k=-\infty}^{\infty}\left(g_{k}-h_{k}\right)=\sum_{k=-\infty}^{\infty}\left(g_{k}-h_{k+1}\right)
$$

We are now ready to describe our approach. Let us take Ramanujan's ${ }_{1} \psi_{1}$ sum [10, Appendix II.29] as an example:

$$
{ }_{1} \psi_{1}\left[\begin{array}{c}
a \\
b
\end{array} ; q, z\right]=\frac{(q, b / a, a z, q / a z ; q)_{\infty}}{(b, q / a, z, b / a z ; q)_{\infty}},
$$


where $|b / a|<|z|<1$. There are many proofs of this identity, see, for example, Hahn [11, Jackson [13, Andrews [2, 3], Ismail [12], Andrews and Askey [4], Berndt 6], and Schlosser [19].

Theorem 2.1. The following is a Gosper pair for Ramanujan's ${ }_{1} \psi_{1}$ sum:

$$
\begin{aligned}
& g_{k}=\frac{a z^{k+1}}{(a z-b)} \frac{(a ; q)_{k}}{(b ; q)_{k}} \\
& h_{k}=\frac{b z^{k}}{(a z-b)} \frac{(a ; q)_{k}}{(b ; q)_{k}} .
\end{aligned}
$$

Step 1. Construct an iteration relation from the closed product form, namely, the right hand side of (2.4).

Substituting $b$ to $b q$ in (2.4), we get

$$
{ }_{1} \psi_{1}\left[\begin{array}{cc}
a & \\
{ }_{b q} & ; q, z
\end{array}\right]=\frac{(q, b q / a, a z, q / a z ; q)_{\infty}}{(b q, q / a, z, b q / a z ; q)_{\infty}} .
$$

Define

$$
f(a, b, z)={ }_{1} \psi_{1}\left[\begin{array}{ll}
a & \\
& ; q, z
\end{array}\right] \text {. }
$$

Comparing the right hand sides of (2.4) and (2.5) gives the following iteration relation (see also [4]):

$$
{ }_{1} \psi_{1}\left[\begin{array}{l}
a \\
b
\end{array} ; q, z\right]=\frac{(1-b / a)}{(1-b)(1-b / a z)}{ }_{1} \psi_{1}\left[\begin{array}{cc}
a & \\
b q
\end{array} ; q, z\right] .
$$

Notice that both sides of the above identity are convergent.

Let $F_{k}(a, b, z)$ and $G_{k}(a, b, z)$ denote the $k$ th terms of the left hand side and the right hand side summations in (2.7), respectively, that is,

$$
F_{k}(a, b, z)=\frac{(a ; q)_{k}}{(b ; q)_{k}} z^{k} \text { and } G_{k}(a, b, z)=\frac{(1-b / a)}{(1-b)(1-b / a z)} F_{k}(a, b q, z) \text {. }
$$

Step 2. Apply the $q$-Gosper algorithm to find a Gosper pair $\left(g_{k}, h_{k}\right)$.

It is essential to observe that $F_{k}(a, b, z)-G_{k}(a, b, z)$ is a basic hypergeometric term. In fact, it can be written as

$$
\left(1-b q^{k}-\frac{1-b / a}{1-b / a z}\right) \frac{(a ; q)_{k}}{(b ; q)_{k+1}} z^{k} .
$$

Now we may employ the $q$-Gosper algorithm with respect to the equation

$$
F_{k}(a, b, z)-G_{k}(a, b, z)=h_{k+1}-h_{k},
$$

and we find a solution

$$
h_{k}=\frac{b z^{k}}{(a z-b)} \frac{(a ; q)_{k}}{(b ; q)_{k}}
$$

which also satisfies the limit condition

$$
\lim _{k \rightarrow \infty} h_{k}=\lim _{k \rightarrow-\infty} h_{k}=0
$$


As far as the verification of (2.7) is concerned, the existence of a solution $h_{k}$ and the limit condition (2.2) would guarantee that the identity holds. Now it takes one more step to compute the Gosper pair:

$$
g_{k}=h_{k}+F_{k}(a, b, z)=\frac{a z^{k+1}}{(a z-b)} \frac{(a ; q)_{k}}{(b ; q)_{k}} .
$$

Step 3. Based on the iteration relation and the limit value, one can verify the summation formula.

From the iteration relation (2.7), we may reduce the evaluation of the bilateral series ${ }_{1} \psi_{1}$ to the special case

$$
f(a, b, z)=\frac{(b / a ; q)_{\infty}}{(b, b / z a ; q)_{\infty}} f(a, 0, z) .
$$

Setting $b=q$ in (2.12), we get

$$
f(a, 0, z)=\frac{(q, q / z a ; q)_{\infty}}{(q / a ; q)_{\infty}} \sum_{k=-\infty}^{\infty} \frac{(a ; q)_{k}}{(q ; q)_{k}} z^{k} .
$$

Invoking the relation (1.1), we see that $1 /(q, q)_{-k}=0$ for any positive integer $k$. Consequently, the above bilateral sum reduces to a unilateral sum. Exploiting the $q$-binomial theorem [10, Appendix II.3]

$$
\sum_{k=0}^{\infty} \frac{(a ; q)_{k}}{(q ; q)_{k}} z^{k}=\frac{(a z ; q)_{\infty}}{(z ; q)_{\infty}}
$$

we get the evaluation

$$
f(a, 0, z)=\frac{(q, a z, q / a z ; q)_{\infty}}{(q / a, z ; q)_{\infty}} .
$$

Hence the identity (2.4) follows from (2.12) and (2.14).

It should be warned that it is not always the case that there is a solution $h_{k}$ to the equation (2.9). If one encounters such a scenario, one still has an alternative to try another iteration relation, as is done for the ${ }_{3} \psi_{3}$ sum in Example 2.4

An Abel pair $\left(A_{k}, B_{k}\right)$ can be easily constructed from a Gosper pair $\left(g_{k}, h_{k}\right)$. Setting

$$
g_{k}=A_{k} B_{k} \text { and } h_{k}=A_{k} B_{k-1} \text {, }
$$

then we see that

$$
\frac{B_{k}}{B_{k-1}}=\frac{g_{k}}{h_{k}} .
$$

Without loss of generality, we may assume that $B_{0}=1$. Iterating (2.16) yields an Abel pair $\left(A_{k}, B_{k}\right)$.

For Ramanujan's ${ }_{1} \psi_{1}$ sum (2.4), we can compute an Abel pair by using the $q$-Gosper algorithm.

Theorem 2.2. The following is an Abel pair for Ramanujan's ${ }_{1} \psi_{1}$ sum:

$$
\begin{aligned}
& A_{k}=\frac{a z}{a z-b} \frac{(a ; q)_{k}}{(b ; q)_{k}}\left(\frac{b}{a}\right)^{k}, \\
& B_{k}=\left(\frac{a z}{b}\right)^{k} .
\end{aligned}
$$


It is routine to verify that $\left(A_{k}, B_{k}\right)$ is indeed an Abel pair for the ${ }_{1} \psi_{1}$ sum. First we have

$$
\begin{aligned}
& B_{k}-B_{k-1}=\left(\frac{a z}{b}-1\right)\left(\frac{a z}{b}\right)^{k-1}, \\
& A_{k}-A_{k+1}=\frac{(1-b / a)}{(1-b)(1-b / a z)} \frac{(a ; q)_{k}}{(b q ; q)_{k}}\left(\frac{b}{a}\right)^{k} .
\end{aligned}
$$

Then the iteration relation (2.7) is deduced from the Abel lemma

$$
\begin{aligned}
& \sum_{k=-\infty}^{\infty} \frac{a z}{a z-b} \frac{(a ; q)_{k}}{(b ; q)_{k}}\left(\frac{b}{a}\right)^{k}\left(\frac{a z}{b}-1\right)\left(\frac{a z}{b}\right)^{k-1} \\
& =\sum_{k=-\infty}^{\infty}\left(\frac{a z}{b}\right)^{k} \frac{(1-b / a)}{(1-b)(1-b / a z)} \frac{(a ; q)_{k}}{(b q ; q)_{k}}\left(\frac{b}{a}\right)^{k} .
\end{aligned}
$$

Next we give some examples for bilateral summations.

Example 2.3. The sum of a well-poised ${ }_{2} \psi_{2}$ series ([10], Appendix II.30):

$$
{ }_{2} \psi_{2}\left[\begin{array}{cc}
b, & c \\
a q / b, & a q / c
\end{array} ; q,-\frac{a q}{b c}\right]=\frac{(a q / b c ; q)_{\infty}\left(a q^{2} / b^{2}, a q^{2} / c^{2}, q^{2}, a q, q / a ; q^{2}\right)_{\infty}}{(a q / b, a q / c, q / b, q / c,-a q / b c ; q)_{\infty}}
$$

where $|a q / b c|<1$.

Write the $k$ th term of the left hand side of (2.18) as

$$
F_{k}(a, b, c)=\frac{(b, c ; q)_{k}}{(a q / b, a q / c ; q)_{k}}\left(-\frac{a q}{b c}\right)^{k} .
$$

Substituting $b$ with $b / q$ in (2.18), we are led to the iteration relation

$$
\sum_{k=-\infty}^{\infty} F_{k}(a, b, c)=\frac{(1-a q / b c)\left(1-a q^{2} / b^{2}\right)}{(1+a q / b c)(1-q / b)(1-a q / b)} \sum_{k=-\infty}^{\infty} F_{k}(a, b / q, c) .
$$

Let

$$
G_{k}(a, b, c)=\frac{(1-a q / b c)\left(1-a q^{2} / b^{2}\right)}{(1+a q / b c)(1-q / b)(1-a q / b)} F_{k}(a, b / q, c) .
$$

Implementing the $q$-Gosper algorithm, we obtain a Gosper pair

$$
\begin{aligned}
& g_{k}=\frac{\left(b^{2} c q^{k}-a q^{2}\right)}{(a q+b c)\left(b q^{k}-q\right)} F_{k}(a, b, c), \\
& h_{k}=\frac{b q\left(c-a q^{k}\right)}{(a q+b c)\left(b q^{k}-q\right)} F_{k}(a, b, c) .
\end{aligned}
$$

The companion Abel pair is given below:

$$
\begin{aligned}
A_{k} & =\frac{(b, c ; q)_{k}\left(b^{2} c q^{k}-a q^{2}\right)}{\left(a q / b, b^{2} c / a q ; q\right)_{k}(a q+b c)\left(-q+b q^{k}\right)}, \\
B_{k} & =\frac{\left(b^{2} c / a q ; q\right)_{k}}{(a q / c ; q)_{k}}\left(-\frac{a q}{b c}\right)^{k} .
\end{aligned}
$$


Noticing that (2.18) is symmetric in $b$ and $c$, we have

$$
\begin{aligned}
& \sum_{k=-\infty}^{\infty} F_{k}(a, b, c)=\sum_{k=-\infty}^{\infty} F_{k}(a, b / q, c / q) \\
& \quad \times \frac{(1-a q / b c)\left(1-a q^{2} / b c\right)\left(1-a q^{2} / b^{2}\right)\left(1-a q^{2} / c^{2}\right)}{(1+a q / b c)\left(1+a q^{2} / b c\right)(1-q / b)(1-q / c)(1-a q / b)(1-a q / c)} .
\end{aligned}
$$

Finally, we can reach (2.18) by iterating (2.22) infinitely many times and by using Jacobi's triple product identity (1.9) as the limit case.

Example 2.4. Bailey's sum of a well-poised ${ }_{3} \psi_{3}$ ([10], Appendix II.31):

$$
{ }_{3} \psi_{3}\left[\begin{array}{ccc}
b, & c, & d \\
q / b, & q / c, & q / d
\end{array} ; q, \frac{q}{b c d}\right]=\frac{(q, q / b c, q / b d, q / c d ; q)_{\infty}}{(q / b, q / c, q / d, q / b c d ; q)_{\infty}},
$$

where $|q / b c d|<1$.

Substituting $d$ with $d / q$ in (2.23), one obtains the iteration relation

$$
\begin{aligned}
{ }_{3} \psi_{3}\left[\begin{array}{ccc}
b, & c, & d \\
q / b, & q / c, & q / d
\end{array} ; q, \frac{q}{b c d}\right] \\
\quad=\frac{(1-q / b d)(1-q / c d)}{(1-q / d)(1-q / b c d)}{ }_{3} \psi_{3}\left[\begin{array}{ccc}
q, & c, & d / q \\
q / b, & q / c, & q^{2} / d
\end{array} ; q, \frac{q^{2}}{b c d}\right] .
\end{aligned}
$$

We remark that this sum is in fact an example for which the $q$-Gosper algorithm does not succeed for the iteration relation derived from a straightforward substitution such as $d \rightarrow d q$ or $d \rightarrow d / q$. Instead, using an idea of Paule [16 of symmetrizing a bilateral summation, we replace $k$ by $-k$ on the left hand side of (2.23) to get

$$
{ }_{3} \psi_{3}\left[\begin{array}{ccc}
b, & c, & d \\
q / b, & q / c, & q / d
\end{array} ; q, \frac{q^{2}}{b c d}\right] .
$$

Let $F_{k}(b, c, d)$ be the average of the $k$ th summands of (2.23) and (2.25), namely,

$$
F_{k}(b, c, d)=\frac{(b, c, d ; q)_{k}}{(q / b, q / c, q / d ; q)_{k}}\left(\frac{q}{b c d}\right)^{k} \frac{1+q^{k}}{2},
$$

and let

$$
G_{k}(b, c, d)=\frac{(1-q / b d)(1-q / c d)}{(1-q / d)(1-q / b c d)} F_{k}(b, c, d / q) .
$$

With regard to $F_{k}(b, c, d)-G_{k}(b, c, d)$, the $q$-Gosper algorithm generates a Gosper pair:

$$
\begin{aligned}
g_{k}= & \frac{b d q^{k+1}+c d q^{k+1}-b c d^{2} q^{k}-q^{2}+d q^{k+1}+b c d q^{k+1}-b c d^{2} q^{2 k}-q^{k+2}}{\left(1+q^{k}\right)(b c d-q)\left(q-d q^{k}\right)} \\
& \times F_{k}(b, c, d), \\
h_{k}= & \frac{d\left(b-q^{k}\right)\left(c-q^{k}\right)}{\left(1+q^{k}\right)(q-b c d)\left(1-d q^{k-1}\right)} F_{k}(b, c, d),
\end{aligned}
$$


which implies the iteration relation (2.24). Invoking the symmetric property of the parameters $b, c$, and $d$, we have

$$
\begin{aligned}
& { }_{3} \psi_{3}\left[\begin{array}{ccc}
b, & c, & d \\
q / b, & q / c, & q / d
\end{array} ; q, \frac{q}{b c d}\right]=\frac{(1-q / b c)\left(1-q^{2} / b c\right)(1-q / b d)\left(1-q^{2} / b d\right)}{(1-q / b)(1-q / c)(1-q / d)(1-q / b c d)} \\
& \times \frac{(1-q / c d)\left(1-q^{2} / c d\right)}{\left(1-q^{2} / b c d\right)\left(1-q^{3} / b c d\right)}{ }_{3} \psi_{3}\left[\begin{array}{ccc}
b / q, & c / q, & d / q \\
q^{2} / b, & q^{2} / c, & q^{2} / d
\end{array} ; q, \frac{q^{4}}{b c d}\right] .
\end{aligned}
$$

The above relation enables us to reduce the summation formula (2.23) to Jacobi's triple product identity.

Example 2.5. A basic bilateral analogue of Dixon's sum [10, Appendix II.32]

$$
\begin{aligned}
& { }_{4} \psi_{4}\left[\begin{array}{cccc}
-q a^{\frac{1}{2}}, & b, & c, & d \\
-a^{\frac{1}{2}}, & a q / b, & a q / c, & a q / d
\end{array} ; q, \frac{q a^{\frac{3}{2}}}{b c d}\right] \\
& =\frac{\left(a q, a q / b c, a q / b d, a q / c d, q a^{\frac{1}{2}} / b, q a^{\frac{1}{2}} / c, q a^{\frac{1}{2}} / d, q, q a ; q\right)_{\infty}}{\left(a q / b, a q / c, a q / d, q / b, q / c, q / d, q a^{\frac{1}{2}}, q a^{-\frac{1}{2}}, q a^{\frac{3}{2}} / b c d ; q\right)_{\infty}},
\end{aligned}
$$

where $\left|q a^{\frac{3}{2}} / b c d\right|<1$.

For the above formula, we may consider the substitution $d \rightarrow d / q$ in (2.29) which suggests the iteration relation

$$
\begin{aligned}
& { }_{4} \psi_{4}\left[\begin{array}{cccc}
-q a^{\frac{1}{2}}, & b, & c, & d \\
-a^{\frac{1}{2}}, & a q / b, & a q / c, & a q / d
\end{array} ; q, \frac{q a^{\frac{3}{2}}}{b c d}\right] \\
& =\frac{(1-a q / b d)(1-a q / c d)\left(1-q a^{\frac{1}{2}} / d\right)}{(1-a q / d)(1-q / d)\left(1-q a^{\frac{3}{2}} / b c d\right)} \\
& \times{ }_{4} \psi_{4}\left[\begin{array}{cccc}
-q a^{\frac{1}{2}}, & b, & c, & d / q \\
-a^{\frac{1}{2}}, & a q / b, & a q / c, & a q^{2} / d
\end{array} ; q, \frac{q^{2} a^{\frac{3}{2}}}{b c d}\right] .
\end{aligned}
$$

Let

$$
F_{k}(a, b, c, d)=\frac{\left(-q a^{\frac{1}{2}}, b, c, d ; q\right)_{k}}{\left(-a^{\frac{1}{2}}, a q / b, a q / c, a q / d ; q\right)_{k}}\left(\frac{q a^{\frac{3}{2}}}{b c d}\right)^{k}
$$

and let

$$
G_{k}(a, b, c, d)=\frac{(1-a q / b d)(1-a q / c d)\left(1-q a^{\frac{1}{2}} / d\right)}{(1-a q / d)(1-q / d)\left(1-q a^{\frac{3}{2}} / b c d\right)} F_{k}(a, b, c, d / q) .
$$

By computation, we obtain a Gosper pair

$$
\begin{aligned}
g_{k}= & \left(\frac{-a b d q^{k+1}-a c d q^{k+1}+q^{2} a^{\frac{3}{2}}+a^{2} q^{k+2}-b c d a^{\frac{1}{2}} q^{k+1}-d a^{\frac{3}{2}} q^{k+1}}{\left(d q^{k}-q\right)\left(1+a^{\frac{1}{2}} q^{k}\right)\left(b c d-a^{\frac{3}{2}} q\right)}\right. \\
& \left.+\frac{b c d^{2} q^{k}+b c d^{2} a^{\frac{1}{2}} q^{2 k}}{\left(d q^{k}-q\right)\left(1+a^{\frac{1}{2}} q^{k}\right)\left(b c d-a^{\frac{3}{2}} q\right)}\right) F_{k}(a, b, c, d), \\
h_{k}= & \frac{d\left(a q^{k}-c\right)\left(a q^{k}-b\right)}{\left(d q^{k-1}-1\right)\left(1+a^{\frac{1}{2}} q^{k}\right)\left(b c d-q a^{\frac{3}{2}}\right)} F_{k}(a, b, c, d) .
\end{aligned}
$$


So the iteration relation (2.30) holds. From the symmetric property of the parameters $b, c$, and $d$, we have

$$
\begin{aligned}
& { }_{4} \psi_{4}\left[\begin{array}{cccc}
-q a^{\frac{1}{2}}, & b, & c, & d \\
-a^{\frac{1}{2}}, & a q / b, & a q / c, & a q / d
\end{array} ; q, \frac{q a^{\frac{3}{2}}}{b c d}\right] \\
& =\frac{(1-a q / b c)\left(1-a q^{2} / b c\right)(1-a q / b d)\left(1-a q^{2} / b d\right)(1-a q / c d)\left(1-a q^{2} / c d\right)}{(1-a q / b)(1-a q / c)(1-a q / d)(1-q / b)(1-q / c)(1-q / d)\left(1-q a^{\frac{3}{2}} / b c d\right)} \\
& \times \frac{\left(1-q a^{\frac{1}{2}} / b\right)\left(1-q a^{\frac{1}{2}} / c\right)\left(1-q a^{\frac{1}{2}} / d\right)}{\left(1-q^{2} a^{\frac{3}{2}} / b c d\right)\left(1-q^{3} a^{\frac{3}{2}} / b c d\right)} \\
& \times{ }_{4} \psi_{4}\left[\begin{array}{cccc}
-q a^{\frac{1}{2}}, & b / q, & c / q, & d / q \\
-a^{\frac{1}{2}}, & a q^{2} / b, & a q^{2} / c, & a q^{2} / d
\end{array} ; q, \frac{q^{4} a^{\frac{3}{2}}}{b c d}\right] .
\end{aligned}
$$

By iteration, it follows that

$$
\begin{aligned}
& { }_{4} \psi_{4}\left[\begin{array}{cccc}
-q a^{\frac{1}{2}}, & b, & c, & d \\
-a^{\frac{1}{2}}, & a q / b, & a q / c, & a q / d
\end{array} ; q, \frac{q a^{\frac{3}{2}}}{b c d}\right] \\
& =\frac{\left(a q / b c, a q / b d, a q / c d, q a^{\frac{1}{2}} / b, q a^{\frac{1}{2}} / c, q a^{\frac{1}{2}} / d ; q\right)_{\infty}}{\left(a q / b, a q / c, a q / d, q / b, q / c, q / d, q a^{\frac{3}{2}} / b c d ; q\right)_{\infty}} H(a),
\end{aligned}
$$

where

$$
H(a)=\sum_{k=-\infty}^{\infty} \frac{\left(-q a^{\frac{1}{2}} ; q\right)_{k}}{\left(-a^{\frac{1}{2}} ; q\right)_{k}} q^{3\left(\begin{array}{c}
k \\
2
\end{array}\right)}\left(-q a^{\frac{3}{2}}\right)^{k} .
$$

Taking $b=-a^{\frac{1}{2}}$ and $c, d \rightarrow \infty$ in (2.34) and by Jacobi's triple product identity (1.9), it can be verified that

$$
H(a)=\frac{(q, a q, q / a ; q)_{\infty}}{\left(q a^{\frac{1}{2}}, q a^{-\frac{1}{2}} ; q\right)_{\infty}}
$$

which leads to (2.29).

Example 2.6. Bailey's very well-poised ${ }_{6} \psi_{6}$-series identity (1.2).

Let us denote the $k$ th term of (1.5) by

$$
\Omega_{k}(a ; b, c, d, e)=\frac{\left(q a^{\frac{1}{2}},-q a^{\frac{1}{2}}, b, c, d, e ; q\right)_{k}}{\left(a^{\frac{1}{2}},-a^{\frac{1}{2}}, a q / b, a q / c, a q / d, a q / e ; q\right)_{k}}\left(\frac{q a^{2}}{b c d e}\right)^{k} .
$$

Set $F_{k}=\Omega_{k}(a ; b, c, d, e)$ and

$$
G_{k}=\Omega_{k}(a q ; b, c, d, e q) \times \frac{a(1-e)(1-a q)(b c-a q)(b d-a q)(c d-a q)}{(1-a)(b-a q)(c-a q)(d-a q)\left(b c d e-a^{2} q\right)} .
$$

By computation, we find the following Gosper pair:

$$
\begin{aligned}
g_{k} & =\frac{a\left(b c d q^{k}-a q\right)\left(1-e q^{k}\right)}{\left(b c d e-a^{2} q\right)\left(1-a q^{2 k}\right)} \Omega_{k}(a ; b, c, d, e), \\
h_{k} & =\frac{\left(e-a q^{k}\right)\left(b c d-a^{2} q^{k+1}\right)}{\left(b c d e-a^{2} q\right)\left(a q^{2 k}-1\right)} \Omega_{k}(a ; b, c, d, e) .
\end{aligned}
$$

We observe that the Abel pair derived from the above Gosper pair coincides with the Abel pair given by Chu [9]. 
Next we give another Gosper pair which leads to a different iteration relation of the ${ }_{6} \psi_{6}$ series by setting $e \rightarrow e / q$ in (1.2), that is,

$$
\Omega(a ; b, c, d, e)=\Omega(a ; b, c, d, e / q) \times \frac{(1-a q / b e)(1-a q / c e)(1-a q / d e)}{(1-a q / e)(1-q / e)\left(1-a^{2} q / b c d e\right)} .
$$

We will see that the above iteration has the advantage that it directly points to the identity (1.8) by taking into account the symmetries in $b, c, d$, and $e$. On the other hand, the Gosper pair does not have a simple expression in this case.

Set $F_{k}=\Omega_{k}(a ; b, c, d, e)$ and

$$
G_{k}=\Omega_{k}(a ; b, c, d, e / q) \times \frac{(1-a q / b e)(1-a q / c e)(1-a q / d e)}{(1-a q / e)(1-q / e)\left(1-a^{2} q / b c d e\right)} .
$$

From the above iteration relation (2.39), we obtain the Gosper pair

$$
\begin{aligned}
g_{k}= & \left(\frac{a^{2} e q^{k+1}+a^{3} q^{2 k+2}-a^{2} q^{2}-a^{2} b e q^{2 k+1}-a^{2} c e q^{2 k+1}-a^{2} d e q^{2 k+1}-b c d e^{2} q^{k}}{\left(b c d e-q a^{2}\right)\left(e q^{k}-q\right)\left(a q^{2 k}-1\right)}\right. \\
& \left.+\frac{a b c e q^{k+1}+a b d e q^{k+1}+a c d e q^{k+1}+a b c d e^{2} q^{3 k}-a b c d e q^{2 k+1}}{\left(b c d e-q a^{2}\right)\left(e q^{k}-q\right)\left(a q^{2 k}-1\right)}\right) \Omega_{k}(a ; b, c, d, e), \\
h_{k}= & \frac{q e\left(b-a q^{k}\right)\left(c-a q^{k}\right)\left(d-a q^{k}\right)}{\left(b c d e-q a^{2}\right)\left(1-a q^{2 k}\right)\left(e q^{k}-q\right)} \Omega_{k}(a ; b, c, d, e) .
\end{aligned}
$$

Since the parameters $b, c, d$, and $e$ are symmetric in (1.2), we obtain

$$
\begin{aligned}
\Omega(a ; b, c, d, e)= & \Omega(a ; b / q, c / q, d / q, e / q) \\
& \times \frac{(1-a q / b c)\left(1-a q^{2} / b c\right)(1-a q / b d)\left(1-a q^{2} / b d\right)}{(1-a q / b)(1-a q / c)(1-a q / d)(1-a q / e)} \\
& \times \frac{(1-a q / b e)\left(1-a q^{2} / b e\right)(1-a q / c d)\left(1-a q^{2} / c d\right)}{(1-q / b)(1-q / c)(1-q / d)(1-q / e)} \\
& \times \frac{(1-a q / c e)\left(1-a q^{2} / c e\right)(1-a q / d e)\left(1-a q^{2} / d e\right)}{\left(1-a^{2} q / b c d e\right)\left(1-a^{2} q^{2} / b c d e\right)\left(1-a^{2} q^{3} / b c d e\right)\left(1-a^{2} q^{4} / b c d e\right)} .
\end{aligned}
$$

Again, the limit value can be given by Jacobi's triple product identity, so that we arrive at (1.2) in view of (2.41).

The following is Shukla's very-well-poised ${ }_{8} \psi_{8}$ summation 21. Note that the relation we aim to verify is not an iteration relation. Instead, we establish the identity based on the observation that the product formula contains the factors in Bailey's ${ }_{6} \psi_{6}$ identity.

Example 2.7. Shukla's very-well-poised ${ }_{8} \psi_{8}$ summation formula:

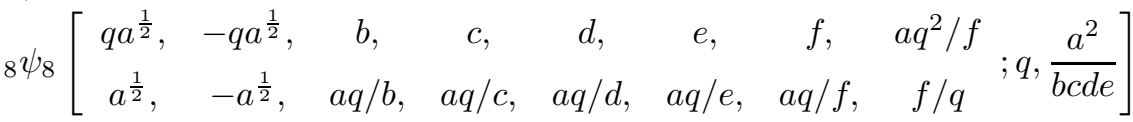

$$
\begin{aligned}
& =\left(1-\frac{(1-b c / a)(1-b d / a)(1-b e / a)}{(1-b q / f)(1-b f / a q)\left(1-b c d e / a^{2}\right)}\right) \times \frac{(1-f / b q)(1-b f / a q)}{(1-f / a q)(1-f / q)} \\
& \times \frac{(q, a q, q / a, a q / b c, a q / b d, a q / b e, a q / c d, a q / c e, a q / d e ; q)_{\infty}}{\left(a q / b, a q / c, a q / d, a q / e, q / b, q / c, q / d, q / e, a^{2} q / b c d e ; q\right)_{\infty}},
\end{aligned}
$$

where $\left|a^{2} / b c d e\right|<1$. 
Observe that the right hand side of (2.42) can be rewritten as

$$
\left(1-\frac{(1-b c / a)(1-b d / a)(1-b e / a)}{(1-b q / f)(1-b f / a q)\left(1-b c d e / a^{2}\right)}\right) \frac{(1-f / b q)(1-b f / a q)}{(1-f / a q)(1-f / q)} \Omega(a ; b, c, d, e),
$$

where $\Omega(a ; b, c, d, e)$ denotes the ${ }_{6} \psi_{6}$ series as given by (1.5).

Let $F_{k}$ denote the $k$ th term of the left hand side of (2.42), namely,

$$
F_{k}=\frac{\left(q a^{\frac{1}{2}},-q a^{\frac{1}{2}}, b, c, d, e, f, a q^{2} / f ; q\right)_{k}}{\left(a^{\frac{1}{2}},-a^{\frac{1}{2}}, a q / b, a q / c, a q / d, a q / e, a q / f, f / q ; q\right)_{k}}\left(\frac{a^{2}}{b c d e}\right)^{k},
$$

and let

$$
\begin{aligned}
G_{k}= & \Omega_{k}(a ; b, c, d, e) \times\left(1-\frac{(1-b c / a)(1-b d / a)(1-b e / a)}{(1-b q / f)(1-b f / a q)\left(1-b c d e / a^{2}\right)}\right) \\
& \times \frac{(1-f / b q)(1-b f / a q)}{(1-f / a q)(1-f / q)},
\end{aligned}
$$

where $\Omega_{k}(a ; b, c, d, e)$ is the $k$ th term of the ${ }_{6} \psi_{6}$ series $\Omega(a ; b, c, d, e)$, as given by (2.37). With the aid of the $q$-Gosper algorithm for $F_{k}-G_{k}$, we find

$$
h_{k}=\frac{f\left(b-a q^{k}\right)\left(c-a q^{k}\right)\left(d-a q^{k}\right)\left(e-a q^{k}\right)}{q^{k-1}(f-q)(f-a q)\left(b c d e-a^{2}\right)\left(1-a q^{2 k}\right)} \Omega_{k}(a ; b, c, d, e) .
$$

Thus (2.42) can be deduced from the telescoping relation (2.3) and the ${ }_{6} \psi_{6}$ formula (1.2).

We now turn to two identities due to Schlosser [20] derived by matrix inverse .

Example 2.8. Let $a, b, c, d, e$, and $u$ be indeterminates. Then

$$
\begin{aligned}
& \sum_{k=-\infty}^{\infty} \frac{1-a q^{2 k}}{(1-a)} \frac{(b, c ; q)_{k}}{(a q / b, a q / c ; q)_{k}} \frac{(d q, e q ; q)_{k}}{(a q / d, a q / e ; q)_{k}}\left(\frac{a^{2}}{b c d e}\right)^{k} \\
& \quad \times\left(1-\frac{(1-d e / a)\left(1-u q^{k}\right)\left(1-a^{2} q^{k} / b c u\right)}{(1-a / b c)\left(1-d q^{k}\right)\left(1-e q^{k}\right)}\right) \\
& =\frac{(q, a q, q / a, a q / b c, a q / b d, a q / b e, a q / c d, a q / c e, a / d e ; q)_{\infty}}{\left(q / b, a q / b, q / c, a q / c, 1 / d, a q / d, 1 / e, a q / e, a^{2} q / b c d e ; q\right)_{\infty}} \cdot \frac{(1-a / c u)(1-b u / a)}{(b-a / c)}
\end{aligned}
$$

provided $\left|a^{2} / b c d e\right|<1$.

As in the preceding example, the right hand side of (2.46) contains the product of the ${ }_{6} \psi_{6}$ sum. We may proceed in the same manner. Write the $k$ th term of the left hand side of (2.46) as $F_{k}$, that is,

$$
\begin{aligned}
F_{k}= & \frac{1-a q^{2 k}}{(1-a)} \frac{(b, c ; q)_{k}}{(a q / b, a q / c ; q)_{k}} \frac{(d q, e q ; q)_{k}}{(a q / d, a q / e ; q)_{k}}\left(\frac{a^{2}}{b c d e}\right)^{k} \\
& \times\left(1-\frac{(1-d e / a)\left(1-u q^{k}\right)\left(1-a^{2} q^{k} / b c u\right)}{(1-a / b c)\left(1-d q^{k}\right)\left(1-e q^{k}\right)}\right),
\end{aligned}
$$

and let

$$
G_{k}=\frac{(1-a / c u)(1-b u / a)(1-a / d e)}{(b-a / c)(1-1 / d)(1-1 / e)} \Omega_{k}(a ; b, c, d, e)
$$


where $\Omega_{k}$ is the same notation as in the preceding example. Employing the $q$-Gosper algorithm with respect to $F_{k}-G_{k}$, we obtain

$$
h_{k}=\frac{\left(b-a q^{k}\right)\left(c-a q^{k}\right)\left(d-a q^{k}\right)\left(e-a q^{k}\right)}{a q^{k}(1-d)(1-e)(a-b c)\left(1-a q^{2 k}\right)} \Omega_{k}(a ; b, c, d, e) .
$$

Then we get the desired identity.

The next example is a ${ }_{8} \psi_{8}$ summation formula which can be verified by using our method. It turns out that the limit identity is a special case of Bailey's ${ }_{6} \psi_{6}$ sum.

Example 2.9. Let $a, b, c$, and $d$ be indeterminates. Let $j$ be an arbitrary integer and $N$ a nonnegative integer. Then

$$
\begin{aligned}
& { }_{8} \psi_{8}\left[\begin{array}{cccccccc}
q a^{\frac{1}{2}}, & -q a^{\frac{1}{2}}, & b, & c, & d q^{j}, & a q^{-j} / c, & a q^{1+N} / b, & a q^{-N} / d \\
a^{\frac{1}{2}}, & -a^{\frac{1}{2}}, & a q / b, & a q / c, & a q^{1-j} / d, & c q^{1+j}, & b q^{-N}, & d q^{1+N}
\end{array} ; q, q\right] \\
& =\frac{(a q / b c, c q / b, d q, d q / a ; q)_{N}}{(c d q / a, d q / c, q / b, a q / b ; q)_{N}} \frac{\left(c d / a, b d / a, c q, c q / a, d q^{1+N} / b, q^{-N} ; q\right)_{j}}{\left(q, c q / b, d / a, d, b c q^{-N} / a, c d q^{1+N} / a ; q\right)_{j}} \\
& \times \frac{(q, q, a q, q / a, c d q / a, a q / c d, c q / d, d q / c ; q)_{\infty}}{(c q, q / c, d q, q / d, c q / a, a q / c, d q / a, a q / d ; q)_{\infty}} .
\end{aligned}
$$

Let $\Lambda(a, b, c, d)$ denote the above ${ }_{8} \psi_{8}$ summation, and let $\Lambda_{k}(a, b, c, d)$ be the $k$ th term of this sum, namely,

$$
\Lambda_{k}(a, b, c, d)=\frac{\left(q a^{\frac{1}{2}},-q a^{\frac{1}{2}}, b, c, d q^{j}, a q^{-j} / c, a q^{1+N} / b, a q^{-N} / d ; q\right)_{k}}{\left(a^{\frac{1}{2}},-a^{\frac{1}{2}}, a q / b, a q / c, a q^{1-j} / d, c q^{1+j}, b q^{-N}, d q^{1+N} ; q\right)_{k}} q^{k} .
$$

Substituting the parameter $b$ with $b / q$ leads to an iteration relation:

$$
\begin{aligned}
\Lambda(a, b, c, d)= & \Lambda(a, b / q, c, d) \frac{(1-a q / b c)(1-c q / b)\left(1-q^{N+1} / b\right)\left(1-a q^{N+1} / b\right)}{(1-q / b)(1-a q / b)\left(1-a q^{N+1} / b c\right)\left(1-c q^{N+1} / b\right)} \\
& \times \frac{\left(1-b d q^{j-1} / a\right)\left(1-d q^{N+1} / b\right)\left(1-b c q^{-N-1} / a\right)\left(1-c q^{j+1} / b\right)}{(1-b d / a q)(1-c q / b)\left(1-d q^{N+j+1} / b\right)\left(1-b c q^{-N+j-1} / a\right)} .
\end{aligned}
$$

Let $F_{k}(a, b, c, d)=\Lambda_{k}(a, b, c, d)$, and let

$$
\begin{aligned}
G_{k}(a, b, c, d)= & \Lambda_{k}(a, b / q, c, d) \times \frac{(1-a q / b c)(1-c q / b)\left(1-q^{N+1} / b\right)\left(1-a q^{N+1} / b\right)}{(1-q / b)(1-a q / b)\left(1-a q^{N+1} / b c\right)\left(1-c q^{N+1} / b\right)} \\
& \times \frac{\left(1-b d q^{j-1} / a\right)\left(1-d q^{N+1} / b\right)\left(1-b c q^{-N-1} / a\right)\left(1-c q^{j+1} / b\right)}{(1-b d / a q)(1-c q / b)\left(1-d q^{N+j+1} / b\right)\left(1-b c q^{-N+j-1} / a\right)} .
\end{aligned}
$$

By the $q$-Gosper algorithm for $F_{k}-G_{k}$, we get

$$
\begin{aligned}
h_{k}= & \Lambda_{k}(a, b, c, d) \times \frac{b\left(1-d q^{N+k}\right)\left(b q^{k}-q^{N+1}\right)\left(1-c q^{k+j}\right)}{q^{k}\left(b c q^{j}-a q^{N+1}\right)\left(b-d q^{N+j+1}\right)(a q-b d)} \\
& \times \frac{\left(d q^{j}-a q^{k}\right)\left(c-a q^{k}\right)\left(b^{2}-a q^{N+2}\right)}{\left(b-c q^{N+1}\right)\left(1-a q^{2 k}\right)\left(1-b q^{k-1}\right)} .
\end{aligned}
$$


The limit identity can be verified as follows:

$$
\begin{aligned}
& \lim _{M \rightarrow \infty} \Lambda\left(a, b q^{-M}, c, d\right) \\
& =\lim _{b \rightarrow \infty} \Lambda(a, b, c, d) \\
& ={ }_{6} \psi_{6}\left[\begin{array}{cccccc}
q a^{\frac{1}{2}}, & -q a^{\frac{1}{2}}, & a q^{-N} / d, & c, & d q^{j}, & a q^{-j} / c \\
a^{\frac{1}{2}}, & -a^{\frac{1}{2}}, & d q^{1+N}, & a q / c, & a q^{1-j} / d, & c q^{1+j}
\end{array} ; q, q^{N+1}\right] \\
& =\frac{\left(q, a q, q / a, d q^{N+1} / c, q^{N-j+1}, c d q^{N+j+1} / a, a q^{1-j} / c d, q^{j+1}, c q / d ; q\right)_{\infty}}{\left(d q^{N+1}, a q / c, a q^{1-j} / d, c q^{j+1}, d q^{N+1} / a, q / c, q^{1-j} / d, c q^{j+1} / a, q^{N+1} ; q\right)_{\infty}} \\
& =\frac{(d q, d q / a ; q)_{N}}{(c d q / a, d q / c ; q)_{N}} \frac{\left(c d / a, c q, c q / a, q^{-N} ; q\right)_{j}}{\left(q, d / a, d, c d q^{1+N} / a ; q\right)_{j}}\left(\frac{d q^{N}}{c}\right)^{j} \\
& \times \frac{(q, q, a q, q / a, c d q / a, a q / c d, c q / d, d q / c ; q)_{\infty}}{(c q, q / c, d q, q / d, c q / a, a q / c, d q / a, a q / d ; q)_{\infty}} .
\end{aligned}
$$

Thus (2.50) can be deduced from (2.52) and (2.54).

\section{The Gosper PAIRs FOR UNILATERAL SUMmations}

The idea of Gosper pairs can be adapted to unilateral summation formulas with a slight modification. We also begin with an iteration relation suggested by a closed product formula which can be stated in the following form:

$$
\sum_{k=0}^{\infty} F_{k}\left(a_{1}, a_{2}, \ldots, a_{n}\right)=\sum_{k=0}^{\infty} G_{k}\left(a_{1}, a_{2}, \ldots, a_{n}\right) .
$$

For the same reason as in the bilateral case, we see that

$$
F_{k}\left(a_{1}, a_{2}, \ldots, a_{n}\right)-G_{k}\left(a_{1}, a_{2}, \ldots, a_{n}\right)
$$

is a basic hypergeometric term so that we can resort to the $q$-Gosper algorithm to solve the following equation:

$$
F_{k}\left(a_{1}, a_{2}, \ldots, a_{n}\right)-G_{k}\left(a_{1}, a_{2}, \ldots, a_{n}\right)=h_{k+1}-h_{k}, \quad k \geq 0 .
$$

We assume that $\sum_{k=0}^{\infty} F_{k}\left(a_{1}, a_{2}, \ldots, a_{n}\right)$ and $\sum_{k=0}^{\infty} G_{k}\left(a_{1}, a_{2}, \ldots, a_{n}\right)$ are convergent. Moreover, we assume that the following limit condition holds:

$$
\lim _{k \rightarrow \infty} h_{k}=h_{0}
$$

A Gosper pair $\left(g_{k}, h_{k}\right)$ is defined by the equations

$$
\begin{aligned}
g_{k}-h_{k} & =F_{k}\left(a_{1}, a_{2}, \ldots, a_{n}\right), \\
g_{k}-h_{k+1} & =G_{k}\left(a_{1}, a_{2}, \ldots, a_{n}\right) .
\end{aligned}
$$

Therefore, the identity (3.1) can be demonstrated by the Gosper pair $\left(g_{k}, h_{k}\right)$ because of the telescoping relation

$$
\sum_{k=0}^{\infty}\left(g_{k}-h_{k}\right)=\sum_{k=0}^{\infty}\left(g_{k}-h_{k+1}\right)
$$

and the limit condition $\lim _{k \rightarrow \infty} h_{k}=h_{0}$. 
Given a Gosper pair it is easy to compute the corresponding Abel pair which implies iteration relation (3.1) by the following unilateral form of the Abel lemma:

$$
\sum_{k=0}^{\infty} A_{k}\left(B_{k}-B_{k-1}\right)=\sum_{k=0}^{\infty} B_{k}\left(A_{k}-A_{k+1}\right) .
$$

Note that we also need the limit condition

$$
\lim _{k \rightarrow \infty} A_{k} B_{k-1}=A_{0} B_{-1}
$$

As will be seen, we will encounter only the case $B_{-1}=0$ due to the fact $1 /(q ; q)_{-1}=$ 0 by (1.1).

The above approach is suitable for many classical unilateral summation formulas including the $q$-Gauss sum [10, Appendix II.8], the $q$-Kummer (Bailey-Daum) sum [10, Appendix II.9], the $q$-Dixon sum [10, Appendix II.13], a $q$-analogue of Watson's ${ }_{3} F_{2}$ sum [10, Appendix II.16], and a $q$-analogue of Whipple's ${ }_{3} F_{2}$ sum [10, Appendix II.18], just to name a few. Here we give only two examples to demonstrate this technique.

Example 3.1. The $q$-Gauss sum

$$
{ }_{2} \phi_{1}\left[\begin{array}{cc}
a, & b \\
& c
\end{array} ; q, \frac{c}{a b}\right]=\frac{(c / a, c / b ; q)_{\infty}}{(c, c / a b ; q)_{\infty}},
$$

where $|c / a b|<1$.

Set

$$
f(a, b, c)={ }_{2} \phi_{1}\left[\begin{array}{lll}
a, & b \\
& c
\end{array} ; q, \frac{c}{a b}\right] .
$$

Write the $k$ th term of (3.7) as

$$
F_{k}(a, b, c)=\frac{(a, b ; q)_{k}}{(q, c ; q)_{k}}\left(\frac{c}{a b}\right)^{k} .
$$

The substitution $c \rightarrow c q$ in $(3.6)$ implies

$$
f(a, b, c)=\frac{(1-c / a)(1-c / b)}{(1-c)(1-c / a b)} f(a, b, c q) .
$$

Let

$$
G_{k}(a, b, c)=\frac{(1-c / a)(1-c / b)}{(1-c)(1-c / a b)} F_{k}(a, b, c q) .
$$

Applying the $q$-Gosper algorithm to $F_{k}(a, b, c)-G_{k}(a, b, c)$, we arrive at a Gosper pair

$$
\begin{aligned}
g_{k} & =\frac{c-a b q^{k}}{c-a b} F_{k}(a, b, c), \\
h_{k} & =\frac{a b\left(1-q^{k}\right)}{c-a b} F_{k}(a, b, c) .
\end{aligned}
$$


So we have an Abel pair

$$
\begin{aligned}
A_{k} & =\frac{\left(1-a b q^{k} / c\right)}{(1-a b / c)} \frac{(a, b ; q)_{k}}{(c, a b q / c ; q)_{k}}, \\
B_{k} & =\frac{(a b q / c ; q)_{k}}{(q ; q)_{k}}\left(\frac{c}{a b}\right)^{k} .
\end{aligned}
$$

We see that identity (3.6) is true because of (3.5) and the limit value $f(a, b, 0)=1$.

Example 3.2. The sum of Rogers' nonterminating very-well-poised ${ }_{6} \phi_{5}$ series $[10$, Appendix II.20]:

$$
\begin{aligned}
& { }_{6} \phi_{5}\left[\begin{array}{cccccc}
a, & q a^{\frac{1}{2}}, & -q a^{\frac{1}{2}}, & b, & c, & d \\
& a^{\frac{1}{2}}, & -a^{\frac{1}{2}}, & a q / b, & a q / c, & a q / d
\end{array} ; q, \frac{a q}{b c d}\right] \\
& =\frac{(a q, a q / b c, a q / b d, a q / c d ; q)_{\infty}}{(a q / b, a q / c, a q / d, a q / b c d ; q)_{\infty}},
\end{aligned}
$$

where $|a q / b c d|<1$.

Let us write

$$
f(a, b, c, d)={ }_{6} \phi_{5}\left[\begin{array}{cccccc}
a, & q a^{\frac{1}{2}}, & -q a^{\frac{1}{2}}, & b, & c, & d \\
& a^{\frac{1}{2}}, & -a^{\frac{1}{2}}, & a q / b, & a q / c, & a q / d
\end{array} ; q, \frac{a q}{b c d}\right] .
$$

Denote the $k$ th term of (3.12) by

$$
F_{k}(a, b, c, d)=\frac{\left(a, q a^{\frac{1}{2}},-q a^{\frac{1}{2}}, b, c, d ; q\right)_{k}}{\left(q, a^{\frac{1}{2}},-a^{\frac{1}{2}}, a q / b, a q / c, a q / d ; q\right)_{k}}\left(\frac{a q}{b c d}\right)^{k} .
$$

The substitution $a \rightarrow a q$ in (3.11) leads to the iteration relation

$$
f(a, b, c, d)=\frac{(1-a q)(1-a q / c d)(1-a q / b c)(1-a q / b d)}{(1-a q / b)(1-a q / c)(1-a q / d)(1-a q / b c d)} f(a q, b, c, d) .
$$

Let

$$
G_{k}(a, b, c, d)=\frac{(1-a q)(1-a q / c d)(1-a q / b c)(1-a q / b d)}{(1-a q / b)(1-a q / c)(1-a q / d)(1-a q / b c d)} F_{k}(a q, b, c, d) .
$$

Then we get a Gosper pair

$$
\begin{aligned}
& g_{k}=\frac{\left(1-a q^{k}\right)\left(q^{k}-a q / b c d\right)}{\left(1-a q^{2 k}\right)(1-a q / b c d)} F_{k}(a, b, c, d), \\
& h_{k}=-\frac{\left(1-q^{k}\right)\left(1-a^{2} q^{k+1} / b c d\right)}{\left(1-a q^{2 k}\right)(1-a q / b c d)} F_{k}(a, b, c, d) .
\end{aligned}
$$

The corresponding Abel pair is as follows:

$$
\begin{aligned}
A_{k} & =\frac{\left(b c d q^{k}-a q\right)}{(b c d-a q)} \frac{\left(b, c, d, a^{2} q^{2} / b c d ; q\right)_{k}}{(a q / b, a q / c, a q / d, b c d / a ; q)_{k}}, \\
B_{k} & =\frac{(a q, b c d / a ; q)_{k}}{\left(q, a^{2} q^{2} / b c d ; q\right)_{k}}\left(\frac{a q}{b c d}\right)^{k} .
\end{aligned}
$$

Therefore, the identity (3.11) is a consequence of the unilateral Abel lemma (3.5) and the limit value $f(0, b, c, d)=1$. 


\section{ACKNOWLEDGMENTS}

We are grateful to George Andrews, Michael Schlosser and Doron Zeilberger for valuable comments.

\section{REFERENCES}

1. S. A. Abramov, P. Paule, and M. Petkovšek, q-Hypergeometric solutions of q-difference equations, Discrete Math. 180 (1998), 3-22. MR1603685 (99f:39001)

2. G. E. Andrews, On Ramanujan's summation of ${ }_{1} \psi_{1}(a, b, z)$, Proc. Amer. Math. Soc. 22 (1969), 552-553. MR0241703 (39:3042)

3. G. E. Andrews, On a transformation of bilateral series with applications, Proc. Amer. Math. Soc. 25 (1970), 554-558. MR0257413(41:2064)

4. G. E. Andrews and R. Askey, A simple proof of Ramanujan's summation of the ${ }_{1} \psi_{1}$, Aequationes Math. 18 (1978), 333-337. MR.522519 (80h:33004)

5. W. N. Bailey, Series of hypergeometric type which are infinite in both directions, Quart. J. Math. (Oxford) 7 (1936), 105-115.

6. B. C. Berndt, Ramanujan's theory of theta-functions, In: Theta Functions from the Classical to the Modern, M. R. Murty, ed., CRM Proceedings and Lecture Notes, 1, Amer. Math. Soc., Providence, 1993, pp. 1-63. MR.1224050 (94m:11054)

7. H. Böing and W. Koepf, Algorithm for q-hypergeometric summation in computer algebra, J. Symbolic Comput. 28 (1999), 777-799. MR1750546 (2001j:33019)

8. W. Y. C. Chen, Q. H. Hou, and Y. P. Mu, Nonterminating basic hypergeometric series and the q-Zeilberger algorithm, arXiv:math.CO/0509281.

9. W. C. Chu, Bailey's very well-poised ${ }_{6} \psi_{6}$-series identity, J. Combin. Theory, Ser. A 113 (2006), 966-979 . MR2244127

10. G. Gasper and M. Rahman, Basic Hypergeometric Series, second ed., Cambridge University Press, Cambridge, 2004. MR2128719 (2006d:33028)

11. W. Hahn, Über Polynome, die gleichzeitig zwei verschiedenen Orthogonalsystemen angehören, Math. Nachr. 2 (1949), 263-278. MR0032858 (11:356f)

12. M. E. H. Ismail, A simple proof of Ramanujan's ${ }_{1} \psi_{1}$ sum, Proc. Amer. Math. Soc. 63 (1977), 185-186. MR0508183 (58:22695)

13. M. Jackson, On Lerch's transcendant and the basic bilateral hypergeometric series ${ }_{2} \psi_{2}$, J. London Math. Soc. 25 (1950), 189-196. MR0036882(12:178f)

14. T. H. Koornwinder, On Zeilberger's algorithm and its q-analogue, J. Comput. Appl. Math. 48 (1993), 91-111. MR1246853 (95b:33011)

15. M. Mohammed and D. Zeilberger, Sharp upper bounds for the orders of the recurrences outputted by the Zeilberger and q-Zeilberger Algorithms, J. Symbolic Comput. 39 (2005), 201-207. MR 2169800

16. P. Paule, Short and easy computer proofs of the Roger-Ramanujan identities and of identities of similar type, Electron. J. Combin. 1 (1994), R10. MR1293400 (95g:11099)

17. P. Paule and M. Schorn, A Mathematica version of Zeilberger's algorithm for proving binomial coefficients identities, J. Symbolic Comput. 20 (1995), 673-698. MR1395420 (97j:39006)

18. A. Riese, A generalization of Gosper's algorithm to bibasic hypergeometric summation, Electron. J. Combin. 3 (1996), R19. MR1394550 (97h:33031)

19. M. Schlosser, Abel-Rothe type generalizations of Jacobi's triple product identity, in "Theory and Applications of Special Functions. A Volume Dedicated to Mizan Rahman" (M. E. H. Ismail and E. Koelink, eds.), Dev. Math. 13 (2005), 383-400. MR.2132472 (2006b:33032)

20. M. Schlosser, Inversion of bilateral basic hypergeometric series, Electron. J. Combin. 10 (2003), R10. MR.1975760 (2004d:33017)

21. H. S. Shukla, A note on the sums of certain bilateral hypergeometric series, Proc. Cambridge Phil. Soc. 55 (1959), 262-266. MR0104839 (21:3590)

22. H. S. Wilf and D. Zeilberger, An algorithmic proof theory for hypergeometric (ordinary and "q") multisum/integral identities, Invent. Math. 108 (1992), 575-633. MR.1163239 (93k:33010) 
Center for Combinatorics, LPMC, Nankai University, Tianjin 300071, P. R. China

E-mail address: ybchen@mail.nankai.edu.cn

Center for Combinatorics, LPMC, Nankai University, Tianjin 300071, P. R. China

E-mail address: chen@nankai.edu.cn

Center for Combinatorics, LPMC, Nankai University, Tianjin 300071, P. R. China

E-mail address: gu@nankai.edu.cn 